\title{
A Simplified Maximum Likelihood Detection for MIMO Systems
}

\author{
Hozun SUNG ${ }^{\dagger}$, Nonmember, Jee Woong KANG ${ }^{\dagger a)}$, Member, and Kwang Bok LEE ${ }^{\dagger \dagger}$, Nonmember
}

SUMMARY In this paper, we propose a simplified Maximum Likelihood (ML) detection scheme for Multiple-Input Multiple-Output (MIMO) system that has much less computational complexity than the conventional ML detection scheme. Simulation results verify that the bit error rate (BER) performance of the proposed scheme is close to that of the ML detection scheme with significant complexity reduction.

key words: MIMO, V-BLAST, ML detector

\section{Introduction}

Next generation wireless communication systems require large system capacity for high data rate and multimedia services. Since the radio frequency is a scarce resource, it is desirable to increase spectral efficiency, and the use of Multiple-Input Multiple-Output (MIMO) systems is one of popular approaches to achieve the goal [1]-[4]. During the last decade, a number of space-time architectures have been developed for MIMO systems. The Vertical Belllaboratories LAyered Space-Time (V-BLAST) system is one of the most promising realizations of MIMO systems [5], [6]. However, the performance of the V-BLAST detection scheme, often called Ordered Successive Interference Cancellation (OSIC) detection scheme [6], [7], is limited due to insufficient receive diversity and imperfect interference cancellation. On the other hand, Maximum Likelihood (ML) detection scheme performs the best, whereas its complexity is excessively high especially for large number of transmit antennas. Although there have been some attempts to compromise the performance and complexity [7]-[9], it is still required to investigate the MIMO detector achieving satisfactory performance with moderate complexity.

In this paper, we propose a simplified ML detection scheme for MIMO systems. In the proposed scheme, several symbol combinations are chosen among all the possible symbol combinations by tentative decisions, and the final decision value is obtained by likelihood test among the chosen symbol combinations. By restricting the number of symbol combinations to be examined, the complexity can be greatly reduced with only slight performance degradation over the conventional ML detection scheme.

Manuscript received April 18, 2005.

Manuscript revised December 14, 2005.

${ }^{\dagger}$ The authors are with Samsung Electronics Co. Ltd., Suwonsi, Korea.

${ }^{\dagger \dagger}$ The author is with the School of Electrical Engineering and INMC, Seoul National University, Seoul, Korea.

a) E-mail: jw75.kang@ samsung.com

DOI: $10.1093 /$ ietcom/e89-b.8.2241

\section{System and Channel Model}

We consider a MIMO system equipped with $N$ transmit antennas and $M(\geq N)$ receive antennas. In the transmitter, the input data stream is demultiplexed into $N$ sub-streams, and they are loaded on $N$ transmit antennas with equal transmit power. The radiated signals from transmit antennas are assumed to experience a rich scattering wireless channel. The baseband equivalent model of the received signal vector at sampling instants can be represented as

$$
\mathbf{r}=\sqrt{P / N} \mathbf{H} \cdot \mathbf{x}+\mathbf{n}
$$

where $\mathbf{r}=\left[r_{1} \ldots r_{m} \ldots r_{M}\right]^{T}$ is an $M \times 1$ received signal vector, $\mathbf{x}=\left[x_{1} \ldots x_{n} \ldots x_{N}\right]^{T}$ is an $N \times 1$ transmit sub-stream vector, $\mathbf{n}=\left[n_{1} \ldots n_{m} \ldots n_{M}\right]^{T}$ is an $M \times 1$ white complex Gaussian noise vector of which entries have zero mean and variance of $\sigma^{2}, P$ is the total transmit energy, and superscript ${ }^{T}$ denotes the transpose operator. $\mathbf{H}=\left[h_{m, n}\right](m=$ $1,2, \ldots, M$ and $n=1,2, \ldots, N)$ is an $M \times N$ channel impulse response matrix, where $h_{m, n}$ denotes the channel impulse response from the $n$-th transmit antenna to the $m$-th receive antenna and is assumed to be an independent and identically distributed complex Gaussian random variable with zero-mean and unit-variance. Every transmit sub-stream $x_{n}$ is assumed to be a symbol in the set of constellation points $\mathbf{S}$ comprised of $C$ elements.

\section{A Simplified ML Detection Scheme}

The optimal ML detector estimates transmit sub-streams by choosing the symbol combination associated with the minimal distance metric among all possible symbol combination:

$$
\hat{\mathbf{x}}_{\mathrm{ML}}=\underset{\mathbf{s}^{N} \in \mathbf{S}^{N}}{\arg \min }\left\|\mathbf{r}-\sqrt{P / N} \mathbf{H} \cdot \mathbf{s}^{N}\right\|
$$

where $\|\mathbf{a}\|$ is the norm of vector $\mathbf{a}$, and $\mathbf{S}^{N}=$ $\left\{\left[s_{1} \ldots s_{n} \ldots s_{N}\right]^{T} \mid s_{n} \in \mathbf{S}, n=1,2, \ldots, N\right\}$ is the set of possible symbol combinations. As shown in (2), the ML detection scheme needs to examine $C^{N}$ symbol combinations. Therefore, the computational complexity increases rapidly with $N$.

To reduce complexity, we propose a simplified ML detection scheme. The basic idea of the proposed scheme is to perform likelihood test over the reduced number of symbol combinations instead of all $C^{N}$ symbol combinations. 
The proposed scheme is operated over the following threestep detection algorithm. In the first step, several 'candidate symbols' are selected for each sub-stream by linear detection method. In the second step, $N$-dimensional symbol combinations for whole sub-streams are chosen based on each candidate symbol. The chosen combinations are called 'candidate symbol combinations.' In the third step, the final decision is made through likelihood tests over the candidate symbol combinations. From now on, we explain the proposed scheme in detail.

3.1 The First Step: Selecting the Candidate Symbols for Each Sub-Stream

In this step, the detector selects $L(1 \leq L \leq C)$ candidate symbols for each sub-stream. Since there are $N$ sub-streams, the total number of candidate symbols is $L N$. The candidate symbols are estimated using linear detector, such as ZeroForcing (ZF) or Minimum Mean-Square-Error (MMSE). In this paper, the MMSE detector is assumed to be employed for better performance. The MMSE nulling matrix $\mathbf{G}$ for the received signal in (1) can be calculated as

$$
\mathbf{G}=\sqrt{P / N} \mathbf{H}^{H} \cdot\left[P / N \mathbf{H} \cdot \mathbf{H}^{H}+\sigma^{2} \mathbf{I}_{M}\right]^{-1},
$$

where superscript ${ }^{H}$ denotes the conjugate transpose operator, and $\mathbf{I}_{M}$ denotes the $M \times M$ identity matrix. Using the nulling matrix, tentative statistic vector $\mathbf{z}=\left[z_{1} \ldots z_{n} \ldots z_{N}\right]^{T}$ can be obtained as

$$
\mathbf{z}=\mathbf{G} \cdot \mathbf{r} \text {. }
$$

The $l$-th $(l=1, \ldots, L)$ closest element of $\mathbf{S}$ to $z_{n}$ is determined to be the $l$-th candidate symbol $\breve{z}_{n, l}$ for $x_{n}$.

$$
\breve{z}_{n, l}=\underset{s}{\arg \min }\left\|z_{n}-s\right\|, s\left\{\begin{array}{c}
\in \mathbf{S} \\
\notin\left\{\breve{z}_{n, 1}, \breve{z}_{n, 2}, \ldots, \breve{z}_{n, l-1}\right\}
\end{array}\right.
$$

3.2 The Second Step: Choosing the Candidate Symbol Combination for Each Candidate Symbol

In this step, the detector chooses an $N$-dimensional candidate symbol combination, which is a tentative decision on $\mathbf{x}$, for each candidate symbol. The candidate symbol combination $\breve{\mathbf{x}}_{n, l}$ corresponding to $\breve{z}_{n, l}$ is chosen in the following manner. Since the detector already estimates $x_{n}$ to be $\breve{z}_{n, l}$, only $N-1$ sub-streams should be estimated to choose $\breve{\mathbf{x}}_{n, l}$. For that purpose, we adopt the interference cancellation and nulling method. First, the interference from $\breve{z}_{n, l}$ is regenerated and subtracted from the received signal vector $\mathbf{r}$ as

$$
\mathbf{r}_{n, l}=\mathbf{r}-\sqrt{P / N} \mathbf{h}_{n} \cdot \breve{z}_{n, l},
$$

where $\mathbf{h}_{n}$ means the $n$-th column of $\mathbf{H}$. Secondly, the tentative estimations of $N-1$ sub-streams are obtained using the MMSE nulling method. In this step, considering that the candidate symbol is subtracted, the MMSE nulling matrix should be re-calculated as

$$
\mathbf{G}_{n}=\sqrt{P / N} \mathbf{H}_{n}^{H} \cdot\left[P / N \mathbf{H}_{n} \cdot \mathbf{H}_{n}^{H}+\sigma^{2} \mathbf{I}_{M}\right]^{-1},
$$

where $\mathbf{H}_{n}$ is an $M \times(N-1)$ sub-matrix of $\mathbf{H}$, given as

$$
\mathbf{H}_{n}=\left[\begin{array}{ll}
\mathbf{h}_{1} \ldots \mathbf{h}_{n-1} & \mathbf{h}_{n+1} \ldots \mathbf{h}_{N}
\end{array}\right] .
$$

Then, by multiplying $\mathbf{r}_{n, l}$ by $\mathbf{G}_{n}$, a modified statistic vector $\mathbf{y}_{n, l}=\left[\begin{array}{lll}y_{n, l, 1} & y_{n, l, 2} \ldots y_{n, l, N-1}\end{array}\right]^{T}$ is obtained as

$$
\mathbf{y}_{n, l}=\mathbf{G}_{n} \cdot \mathbf{r}_{n, l},
$$

from which tentative estimations of $N-1$ sub-streams are obtained as

$$
\breve{y}_{n, l, i}=Q\left(y_{n, l, i}\right), \quad i=1,2, \ldots, N-1
$$

where $Q($.$) denotes the slicing operation associated with a$ modulation scheme. Finally, by reconstructing the estimated values in (10) and the corresponding candidate symbol, a candidate symbol combination $\breve{x}_{n, l}$ is given as

$$
\breve{\mathbf{x}}_{n, l}=\left[\begin{array}{llll}
\breve{y}_{n, l, 1} & \breve{y}_{n, l, 2} \ldots \breve{y}_{n, l, n-1} \quad \breve{z}_{n, l} \quad \breve{y}_{n, l, n} \ldots \breve{y}_{n, l, N-1}
\end{array}\right]^{T}
$$

These processes are repeated for all candidate symbols, and as a result, $L N$ candidate symbol combinations are obtained.

\subsection{The Third Step: Determining the Final Decision}

In this step, the final decision value is determined by likelihood test as follows.

$$
\hat{\mathbf{x}}_{\text {proposed }}=\underset{\breve{\mathbf{x}}_{n, l}}{\arg \min }\left\|\mathbf{r}-\sqrt{P / N} \mathbf{H} \cdot \breve{\mathbf{x}}_{n, l}\right\|
$$

This step performs the same operation as in (2), except that likelihood tests are performed over only candidate symbol combinations instead of all possible symbol combinations. It is obvious that the performance of the proposed scheme gets close to that of the ML detection scheme, as more symbol combinations are examined in (12). Since the number of candidate symbol combinations is proportional to $L$, it can be deduced that larger $L$ results in better performance. However, larger $L$ also leads to more computational complexity. This tradeoff will be discussed in the next section.

\subsection{Computational Complexity}

In this subsection, we show the computational complexity of the proposed, ML and OSIC detection schemes. Table 1 compares the number of required multiplications for each scheme. The complexities of the proposed and OSIC schemes are shown to increase in the same order with the number of antennas. On the other hand, the complexity of the ML scheme increases exponentially with the number of transmit antennas.

The above statement can be verified by some examples in Table 2. As $L$ changes from 1 to 4 , the complexity of the proposed scheme is shown to increase. However the complexity for $L=4$ is only $30 \%$ larger than that for $L=1$ in 
Table 1 The number of required multiplications.

\begin{tabular}{|c|c|c|c|}
\hline Scheme & The proposed & ML & OSIC \\
\hline $\begin{array}{c}\text { The first } \\
\text { step }\end{array}$ & $M^{3}+2 N M^{2}+N M+C N$ & & \\
\hline $\begin{array}{l}\text { The second } \\
\text { step }\end{array}$ & $\begin{aligned} N M^{3}+ & \left(2 N^{2}-2 N\right) M^{2} \\
& +L N^{2} M\end{aligned}$ & & \\
\hline $\begin{array}{c}\text { The third } \\
\text { step }\end{array}$ & $\left(L N^{2}+L N\right) M$ & & \\
\hline Total & $\begin{array}{c}(N+1) M^{3}+2 N^{2} M^{2} \\
+\left(2 L N^{2}+L N+N\right) M+C N\end{array}$ & $C^{N}(N+1) M$ & $\begin{array}{c}(N+1) M^{3} \\
+(3 / 2) N^{2} M \\
+((7 / 2) N-1) M-1 \\
\end{array}$ \\
\hline $\begin{array}{l}\text { Total } \\
(N=M)\end{array}$ & $\begin{array}{l}3 N^{4}+(2 L+1) N^{3} \\
+(L+1) N^{2}+C N\end{array}$ & $C^{N}(N+1) N$ & $\begin{aligned} & N^{4}+(5 / 2) N^{3} \\
+ & (7 / 2) N^{2}-N-1\end{aligned}$ \\
\hline
\end{tabular}

Table 2 The number of required multiplications (Examples-QPSK modulation).

\begin{tabular}{|c||c|c|c|c|c|c|}
\hline \multicolumn{1}{|c||}{ The Number } & \multicolumn{4}{c|}{ The proposed } & \multirow{2}{*}{ ML } & \multirow{2}{*}{ OSIC } \\
\cline { 2 - 6 } of Antennas & $L=1$ & $L=2$ & $L=3$ & $L=4$ & & \\
\hline \hline$N=M=4$ & 1008 & 1122 & 1296 & 1440 & 5120 & 467 \\
\hline$N=M=6$ & 4632 & 5100 & 5568 & 6036 & 172032 & 1955 \\
\hline
\end{tabular}

the case of $N=M=6$. According to the table, the computational complexity of the proposed scheme with $L=4$ is about 3 times that of the OSIC detection scheme regardless of the number of antennas. The computational complexity of the proposed scheme is about $20-28 \%$ of that of ML detection scheme for $N=M=4$, and only $2.7-3.5 \%$ for $N=M=6$.

\section{Numerical Results}

In this section, simulation results of the proposed scheme are presented in terms of the bit error rate (BER) performance. For comparison, the results of the ML and OSIC detection scheme are also given. We assume that data is QPSK-modulated.

Figure 1 shows how the BER performance of each scheme varies with the average SNR, $P / \sigma^{2}$, when $N=M=$ 4 . In the case of $L=1$, the proposed scheme provides $1.8 \mathrm{~dB}$ SNR gain over the OSIC scheme for achieving BER of $10^{-3}$. As $L$ increases, BER curve of the proposed scheme is shown to approach that of the ML scheme. When $L=2$, the SNR gap is within $1.5 \mathrm{~dB}$ for any BER region, and when $L=4$, the gap is negligibly small. Figure 2 shows the BER performance in the case of $N=M=6$. Overall performance tendency is similar to the case of $N=M=4$. Although the performance difference between the ML scheme and the proposed scheme is larger compared to the case of $N=M=4$, the SNR gap is only $1.5 \mathrm{~dB}$, when $L=4$.

\section{Conclusions}

In this paper, we proposed a simplified ML detection scheme for MIMO systems. By restricting the number of

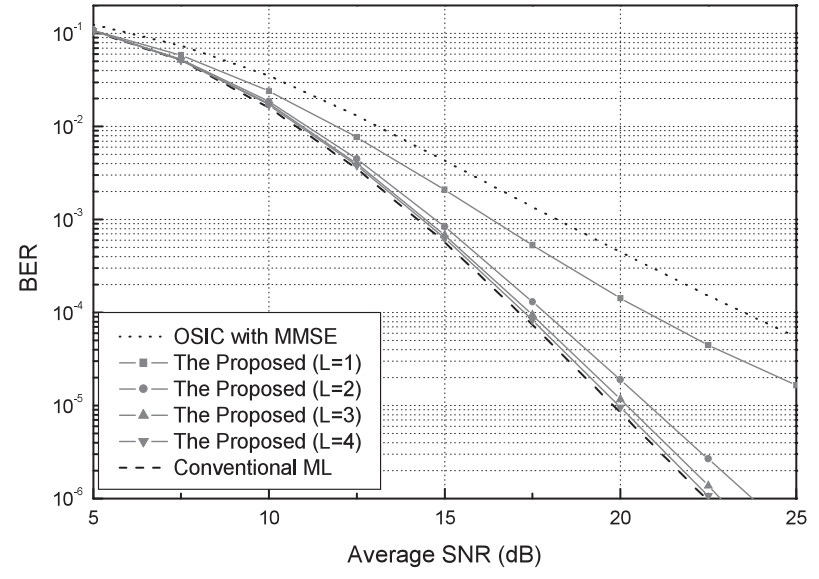

Fig. 1 BER versus average SNR ( $N=M=4$ and QPSK modulation).

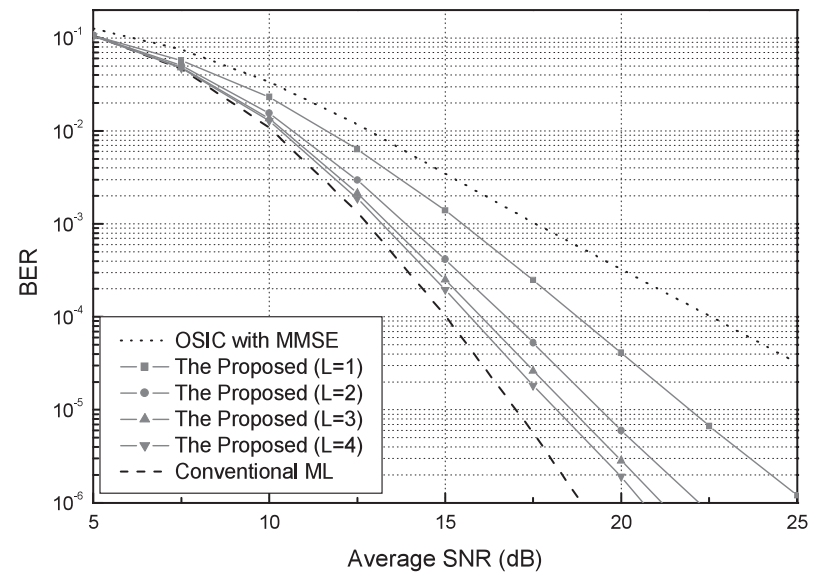

Fig. 2 BER versus average SNR ( $N=M=6$ and QPSK modulation).

symbol combinations to be examined, we can achieve great computational load reduction, especially for the case of large number of antennas. The performance loss is observed to be not significant when the number of candidate symbol combinations is 4 .

\section{Acknowledgments}

This work was supported by National Research Laboratory (NRL) Program.

\section{References}

[1] G.J. Foschini, "Layered space-time architecture for wireless communication in a fading environment when using multiple antennas," Bell Labs Tech. J., vol.1, pp.41-59, Autumn 1996.

[2] G.J. Foschini and M.J. Gans, "On limits of wireless communications in a fading environment when using multiple antennas," Wirel. Pers. Commun., vol.6, pp.311-335, 1998.

[3] G.G. Raleigh and J.M. Cioffi, "Spatio-temporal coding for wireless communications," IEEE Trans. Commun., vol.46, no.3, pp.357-366, March 1998.

[4] V. Tarokh, N. Seshadri, and A.R. Calderbank, "Space-time codes for high data rate wireless communications: Performance criterion and code construction," IEEE Trans. Inf. Theory, vol.44, no.2, pp.744- 
765, March 1998.

[5] P.W. Wolniansky, G.J. Foschini, G.D. Golden, and R.A. Valenzuela, "V-BLAST: An architecture for achieving very high data rates over the rich-scattering wireless channel," Proc. ISSSE-98, pp.295-300, Pisa, Italy, Oct. 1998.

[6] G.J. Foschini, G.D. Golden, R.A. Valenzuela, and P.W. Wolniansky, "Simplified processing for high spectral efficiency wireless communication employing multi-element arrays," IEEE J. Sel. Areas Commun., vol.17, pp.1841-1852, Nov. 1999.

[7] X. Li, H.C. Huang, A. Lozano, and G.J. Foschini, "Reduced- complexity detection algorithms for system using multi-element arrays," Proc. GLOBECOM 2000, pp.1072-1076, San Francisco, CA Nov. 2000

[8] W.J. Choi, R. Negi, and J.M. Cioffi, "Combined ML and DFE decoding for the V-BLAST system," Proc. IEEE International Conference on Communications 2000, pp.1243-1248, New Orleans, LA, June 2000.

[9] W.J. Choi, K.W. Cheong, and J.M. Cioffi, "Iterative soft interference cancellation for multiple antenna systems," Proc. WCNC 2000, pp.304-309, Chicago, IL, Sept. 2000. 